D. Meingafo Flutes 350

E. Nggwals, Managing Sub-Moieties and Manpower 355

Bibliography

Index

\title{
Maps, Figures, and Tables
}

MAPS

1. Languages of the Sepik Basin

2. The Ilahita Arapesh and Adjacent Groups 55

3. Ilahita Village

FIGURES

1. Selected Members of the Torricelli

Language Phylum

19

2. Ward-based Primary Relations

58

3. $\mathrm{Ndu} /$ Nukuma Language Classification

73

4. Transmission of Nungwahim's Magic

99

5. Sister-Exchange Default

102

6. Affinal Discord

107

7. Schema of Ward Inter-marriages

117

8A. Relationships in Selembungambel Hamlet

128

8B. Selembungambel Hamlet

129

9. Cross-Relative Name Bestowal

138

10. Lineal-Collateral Name Transmission

11. Returning a Name

146

12. Masculine Name Transmission

13. A Conflict of Interest

14. Ililip Clan Segmentations

15A. Relationships in Auwi Colony 178

15B. Auwi Colony

16A. Relationships in Ambitemba Camp 200

16B. Ambitemba Camp 201

17. Scheme of the Ilahita Dual Organization 213

18. Initiation Classes 228

19. Atitapwin's Adoption 238 
20. Sub-Moiety Yam Competition 246

21. Transmission of Pig Magic 273

22. Umbaisi Promotion 283

23. The Dual Organization of Ilahita Main Wards 298

24. Meingafo Music 352

TABLES

1. Cash-Crop Production, 1970-71 41

2. Military Alliances in 1940

3. Ilahita Population Dynamics, 1880-1940 72

4. Ilahita Ward Populations, $1969 \quad 92$

5. Inter-Ward Marriages, 1969

6. Residence After Marriage 98

7. Extant Sister-Exchange Marriages, 1969

8. Inter-Ward Marriage Reciprocity 111

9. Ward Hierarchy 114

10. Distribution of Hamlets 126

11. Clans of Ilahita $\quad 167$

12. Clan/Ward Variation $\quad 169$

13. Male Sibling Groups 180

14. Sibling Group Dispersal 181

15. Ilahita Camps 202

16. Ilahita Moieties 220

17. Ilahita Sub-Moieties 224

18. Initiation Class Membership 235

19. Ritual Corporateness of Siblings 237

20. Ilifalemb Moieties and Clans 293 
